\title{
Magnetoelastic anisotropy in NdFeB permanent magnets
}

\author{
C. H. de Groot ${ }^{a)}$ \\ Van der Waals-Zeeman Institute, University of Amsterdam, Valckenierstraat 65, 1018 XE Amsterdam, \\ The Netherlands \\ Kees de Kort \\ Philips Research Laboratories, Professor Holstlaan 4, 5656 AA Eindhoven, The Netherlands
}

(Received 27 October 1998; accepted for publication 11 March 1999)

\begin{abstract}
The influence of magnetoelastic effects on the anisotropy in $\mathrm{NdFeB}$ permanent magnets has been investigated. A relation between the magnetostriction coefficients and the magnetoelastic anisotropy is derived for crystals with tetragonal symmetry. Transmission electron microscopy experiments show that stress and strain are present at the boundaries of the $\mathrm{Nd}_{2} \mathrm{Fe}_{14} \mathrm{~B}$ grains. The origin of the stress is discussed and an order-of-magnitude calculation of its effect on the anisotropy is provided for thermally induced stress. The calculations are in good agreement with the measured influence of stress on coercivity. Both experiment and calculations show that homogeneous stresses have only a small influence on the coercivity. (C) 1999 American Institute of Physics.
\end{abstract}

[S0021-8979(99)04212-7]

\section{INTRODUCTION}

To improve the hard-magnetic properties of $\mathrm{Nd}_{2} \mathrm{Fe}_{14} \mathrm{~B}$-based permanent magnets, numerous elements have been added. ${ }^{1}$ These include low-melting metals (M) like $\mathrm{Al}, \mathrm{Cu}, \mathrm{Ga}$, and $\mathrm{Sn}$, which improve corrosion resistance and increase coercivity by forming intergranular phases other than the Nd-rich phase $\left(\sim \mathrm{Nd}_{80} \mathrm{Fe}_{20}\right)$. The most important intergranular phase formed by the $\mathrm{M}$ elements is the socalled $\delta$ phase, with composition $\mathrm{Nd}_{6} \mathrm{Fe}_{13-x} \mathrm{M}_{1+x} .{ }^{2}$ The increase in coercivity has been attributed to the better wettability of the $\delta$ phases compared to the Nd-rich phase or to the suppression of the formation of soft-magnetic phases. In an article on DyGa addition, we have shown that annealing below $640{ }^{\circ} \mathrm{C}$ leads to the formation of the $\delta$ phase and at the same time to an increase of coercivity from just over 300 to $1200 \mathrm{kA} / \mathrm{m}^{3}{ }^{3}$ From the temperature dependence of the coercivity (using Kronmüller's model ${ }^{4}$ ), we have deduced that this is not due to the suppression of the formation of softmagnetic phases. It seems also unlikely that the increased wettability can account for such a large difference. Therefore, we study in this article the influence of magnetoelastic effects on the anisotropy and coercivity of $\mathrm{NdFeB}$ permanent magnets. Previous investigations of Ramesh ${ }^{5}$ have addressed the same issue. However, this article will show that his assessment of the influence of stress on coercivity is basically incorrect, resulting in a stress-related anisotropy several orders of magnitude larger than the results presented here.

The article is organized as follows: in Sec. II, we derive an expression for the magnetoelastic anisotropy constant for a compound with tetragonal symmetry. In Sec. III, transmission electron microscopy (TEM) measurements are shown which reveal that $\mathrm{Nd}_{2} \mathrm{Fe}_{14} \mathrm{~B}$ grains are stressed near their

\footnotetext{
${ }^{a)}$ Present address: Francis Bitter Magnet Laboratory, Massachusetts Institute of Technology, 170 Albany Street, Cambridge, MA. Electronic mail: grootc@mit.edu
}

boundaries. The origin of the stress will be discussed and the thermal expansion of the various phases will be determined. In Sec. IV, calculations will be performed relating the induced stress to the coercivity. In Sec. V, the main conclusions are presented.

\section{MAGNETOELASTIC ANISOTROPY}

In many textbooks, the relation between the magnetoelastic anisotropy and the magnetostriction for crystals with cubic symmetry is given, $K_{\text {m.e. }}=\frac{3}{2} \lambda_{s} \sigma$, with $\sigma$ the external stress and $\lambda_{s}$ the average spontaneous magnetostriction. ${ }^{6}$ For lower symmetries the situation is more complicated and the magnetization-dependent magnetostrictions must be taken into account. The compound $\mathrm{Nd}_{2} \mathrm{Fe}_{14} \mathrm{~B}$ has $P 4_{2} / \mathrm{mnm}$ symmetry, which means that it has six independent stiffness coefficients and the same number of magnetoelastic terms in the Hamiltonian. Because in aligned magnets the stress is expected to be along a crystallographic axis, we neglect the trigonal distortions, which leaves only three magnetoelastic terms. The one-ion magnetoelastic Hamiltonian $H_{\text {m.e. }}$ is now given by $^{7}$

$$
H_{\text {m.e. }}=-\left(B^{\alpha 1} \epsilon^{\alpha 1}+B^{\alpha 2} \epsilon^{\alpha 2}\right) O_{2}^{0}-B^{\gamma} \epsilon^{\gamma} O_{2}^{2} .
$$

In this expression the $\epsilon^{\mu}$, s are the symmetrized external strains, the $B^{\mu}$ 's the associated magnetoelastic coefficients as given in Ref. 7, and $O_{2}^{0}$ and $O_{2}^{2}$ the Stevens operator equivalents. This results in the following Hamiltonian:

$$
H=H_{\text {ex }}+H_{\text {m.c. }}+H_{\text {m.e. }} \text {. }
$$

Because the exchange interaction is very large, the magnetoelastic Hamiltonian may be considered as a first-order perturbation to the exchange Hamiltonian. It can, therefore, be treated in the same way as the magnetocrystalline Hamiltonian; the diagonalization is reduced to a transformation of the perturbation to the correct coordinate system. ${ }^{8}$ In other words, the Stevens operator equivalents which are defined 
with respect to the crystallographic coordinate system, have to be transformed into the exchange-field-related coordinates, which are defined with respect to the direction of magnetization. This transformation ${ }^{9}$ leads to the following expression for the magnetoelastic energy $E_{\text {m.e. }}$ :

$$
\begin{aligned}
E_{\text {m.e. }}= & \frac{3 \cos ^{2} \theta-1}{2}\left(B^{\alpha 1} \epsilon^{\alpha 1}+B^{\alpha 2} \epsilon^{\alpha 2}\right) \overline{\left\langle O_{2}^{0}\right\rangle}+\frac{\sin ^{2} \theta}{2} \\
& \times \cos (2 \phi) B^{\gamma} \epsilon^{\gamma} \overline{\left\langle O_{2}^{0}\right\rangle}+\cdots \overline{\left\langle O_{2}^{1}\right\rangle}+\cdots \overline{\left\langle O_{2}^{2}\right\rangle},
\end{aligned}
$$

where $\overline{\left\langle O_{n}^{m}\right\rangle}$ is the thermal average of the expectation value. For the first-order magnetoelastic anisotropy constant $K_{\text {m.e. }}$, we are only interested in the $\cos ^{2} \theta$ dependence of the firstorder perturbation $\overline{\left\langle O_{2}^{0}\right\rangle}$. Thus, $K_{\text {m.e. }}$ simplifies to

$K_{\text {m.e. }}=\frac{3}{2}\left(B^{\alpha 1} \epsilon^{\alpha 1}+B^{\alpha 2} \epsilon^{\alpha 2}\right) \overline{\left\langle O_{2}^{0}\right\rangle}-\frac{1}{2} \cos (2 \phi) B^{\gamma} \epsilon^{\gamma} \overline{\left\langle O_{2}^{0}\right\rangle}$,

where $\phi$ is the angle between the strain direction and the in-plane component of the magnetization. The orthorhombic distortion $B^{\gamma} \epsilon^{\gamma}$ breaks the tetragonal symmetry, which for an easy-axis system leads to a difference in hard-magnetization directions perpendicular to the $c$ axis. Because the appropriate anisotropy for magnetization reversal is given by the path of magnetization reversal with the lowest-energy difference, the anisotropy should be minimized with respect to $\phi$. Depending on the sign of $B^{\gamma} \epsilon^{\gamma}, \phi=0^{\circ}$ or $90^{\circ}$, which means that the orthorhombic distortion always lowers the easy-axis anisotropy no matter whether the stress is tensile or compressive.

The magnetoelastic coefficients may be expressed in terms of the stiffness coefficients through the minimization of the free energy, ${ }^{7}$

$$
\begin{aligned}
& B^{\alpha 1} \overline{\left\langle O_{2}^{0}\right\rangle}=C^{\alpha 1} \lambda^{\alpha 1}+C^{\alpha 12} \lambda^{\alpha 2}, \\
& B^{\alpha 2} \overline{\left\langle O_{2}^{0}\right\rangle}=C^{\alpha 2} \lambda^{\alpha 2}+C^{\alpha 12} \lambda^{\alpha 1}, \\
& B^{\gamma \overline{\left\langle O_{2}^{0}\right\rangle}}=C^{\gamma} \lambda^{\gamma},
\end{aligned}
$$

where $C^{\mu}$ are the symmetrized stiffness constants and $\lambda^{\mu}$ are the symmetrized magnetostriction coefficients. We emphasize that these are magnetization-dependent magnetostriction coefficients and not the spontaneous magnetostrictions resulting from the exchange contribution. The latter arises from the two-ion interaction term in the Hamiltonian and are already included in the measurements of the magnetocrystalline anisotropy. The symmetrized magnetostrictions can be expressed in the Cartesian strains, which can be measured experimentally. Defining $\lambda_{M l}$ as the strain along $l$ for a given magnetization $M$, the magnetostriction coefficients are given by

$$
\begin{aligned}
& \lambda^{\alpha 1}=\frac{1}{\sqrt{3}}\left(2 \lambda_{c a}-\lambda_{a a}-\lambda_{b a}+\lambda_{c c}-\lambda_{a c}\right), \\
& \lambda^{\alpha 2}=\frac{2}{\sqrt{6}}\left(\lambda_{c c}-\lambda_{a c}+\frac{1}{2}\left(\lambda_{a a}+\lambda_{b a}\right)-\lambda_{c a}\right), \\
& \lambda^{\gamma}=\lambda_{a a}-\lambda_{b a} .
\end{aligned}
$$

TABLE I. Elastic properties of $\mathrm{Nd}_{2} \mathrm{Fe}_{14} \mathrm{~B}, \mathrm{Nd}$, and stainless steel and magnetoelastic properties of $\mathrm{Nd}_{2} \mathrm{Fe}_{14} \mathrm{~B}$. The magnetostriction coefficients are calculated from the strains at $15 \mathrm{~T}$ as measured by algarabel et al. ${ }^{\mathrm{a}}$

$$
\begin{array}{ll}
E(\Phi)=150 \mathrm{GJ} / \mathrm{m}^{3} & v(\Phi)=0.21 \\
E(\mathrm{Nd})=41 \mathrm{GJ} / \mathrm{m}^{3} & \lambda_{\alpha 1}=-87 \times 10^{-6} \\
E(\mathrm{ss})=200 \mathrm{GJ} / \mathrm{m}^{3} & \lambda_{\alpha 2}=27 \times 10^{-6}
\end{array}
$$

${ }^{a}$ See Ref. 10

For an isotropic crystal, the stiffness constants may be expressed in terms of the Young's modulus $E$ and the shear modulus $G$ or the Poisson ratio $v=E / 2 G-1$ :

$$
\begin{aligned}
& C^{\alpha 1}=\frac{2 E}{1-v-2 v^{2}}, \\
& C^{\alpha 2}=C^{\gamma}=E /(1+v), \\
& C^{\alpha 12}=0 .
\end{aligned}
$$

Because $\mathrm{Nd}_{2} \mathrm{Fe}_{14} \mathrm{~B}$ has a very large magnetocrystalline anisotropy, a large external field must be applied to force the magnetization along a certain direction. Algarabel et al. ${ }^{10}$ have measured the strains, $\lambda_{c a}, \lambda_{c c}$, etc., except for $\lambda_{a b}$, in fields up to $15 \mathrm{~T}$. To calculate the magnetostrictions coefficients $\lambda^{\alpha 1}$ and $\lambda^{\alpha 2}$, we must assume that $\lambda_{a b}=\lambda_{a a}$, and therefore, neglect the orthorhombic distortion. The results from these calculations are collected in Table I.

\section{EXPERIMENTAL RESULTS}

The NdFeB magnets were processed using the liquid phase sintering method. $\mathrm{Ga}$ or $\mathrm{Cu}$ was added either in the ingot or as DyGa powder after the ball milling. The magnets were aligned by a $9 \mathrm{~T}$ pulsed field and after pressing, sintered to atomic density at $1090-1120^{\circ} \mathrm{C}$. The magnets were subsequently annealed at either $600{ }^{\circ} \mathrm{C}$ (low coercive, no $\delta$ phase) or $650{ }^{\circ} \mathrm{C}$ (high coercive, presence of $\delta$ phase). The composition of the magnets is $\mathrm{Nd}_{14.2} \mathrm{Fe}_{78.6} \mathrm{~B}_{7.2}$ with up to 1 at. \% of $\mathrm{Fe}$ replaced by $\mathrm{Cu}$ or $\mathrm{Ga}$ or with $3-5$ wt \% DyGa added. The magnetic properties were measured in a vibrating-sample magnetometer [(VSM), Oxford Instruments]. For structural analysis, the samples were cut perpendicular to the $c$ axis. Phase identification was performed by electron-probe microanalysis. The slices of the magnet were thinned by ion-beam milling before TEM examination.

Experimental results of TEM micrographs are shown in Fig. 1. Half circles with different gray scale than the background are visible at the boundaries of the $\mathrm{Nd}_{2} \mathrm{Fe}_{14} \mathrm{~B}(\Phi)$ grains with all intergranular phases, including the $\delta$ phase. These circles are present independent of coercivity and anneal history of the magnet and indicate the presence of stress on the $\Phi$ grains. This stress changes the lattice parameters of the $\mathrm{Nd}_{2} \mathrm{Fe}_{14} \mathrm{~B}$ structure, and hence, the Bragg reflections of the transmission through the $\mathrm{Nd}_{2} \mathrm{Fe}_{14} \mathrm{~B}$ film. Furthermore, the circles indicate that the stress is rather local and expands radially from a point at the grain boundary. The penetration depth of the stress is of the order of $200 \mathrm{~nm}$. It should be remarked that there are no interfacial defects visible in the $\mathrm{Nd}_{2} \mathrm{Fe}_{14} \mathrm{~B}$ grains and the TEM patterns indicate elastic deformations. In contrast, the Nd-rich phase is always multi- 

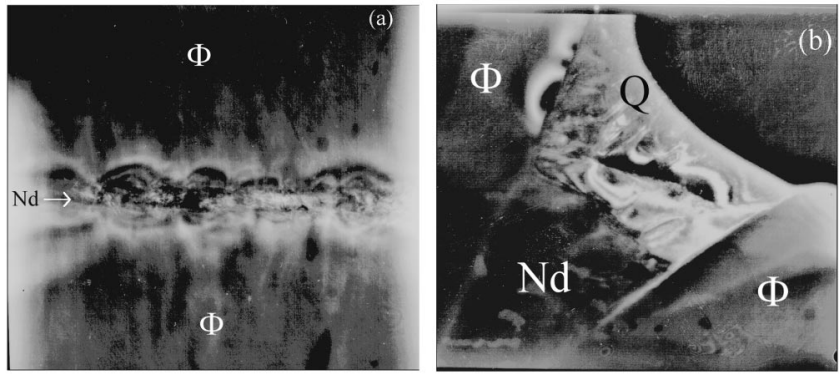

FIG. 1. TEM micrographs of a low-coercive NdFeB magnet with 5\% DyGa. Contrast circles due to stress are visible in the $\Phi$ grains (a) along the grain boundary with a thin layer of Nd-rich phase between the two grains and (b) at a triple point with the $\mathrm{Nd}_{3} \mathrm{Ga}_{2}(Q)$ phase in the top-left corner. Scale: 1 $\mathrm{cm}=400 \mathrm{~nm}$

crystalline and full of defects. The repetition in the stress circles in Fig. 1(a) may be related to a well-known feature of liquid phase sintering called necking. This causes the thickness of the Nd-rich intergranular layer between two $\mathrm{Nd}_{2} \mathrm{Fe}_{14} \mathrm{~B}$ grains to show a periodical variation, which may be reflected in the periodical variation of the stress in the $\mathrm{Nd}_{2} \mathrm{Fe}_{14} \mathrm{~B}$ grains.

The stress is most naturally explained by the differences in thermal expansion of the various phases. The anomalous behavior of the thermal expansion of $\mathrm{Nd}_{2} \mathrm{Fe}_{14} \mathrm{~B}$ forms the basis for these differences. Figure 2 shows the thermal expansion of a $\mathrm{NdFeB}$ permanent magnet (with a small amount of $\mathrm{Cu}$ ), along the $a$ and $c$ axis. As over 90 vol \% of the magnet consists of $\mathrm{Nd}_{2} \mathrm{Fe}_{14} \mathrm{~B}$, the expansion is identical to that measured on aligned $\mathrm{Nd}_{2} \mathrm{Fe}_{14} \mathrm{~B}$ powder. ${ }^{11} \mathrm{~A}$ large spontaneous magnetostriction, especially in the $a$ direction gives an expansion with decreasing temperature below the Curie temperature $\left(312^{\circ} \mathrm{C}\right)$. The thermal expansion of the intergranular $\mathrm{Nd}_{6} \mathrm{Fe}_{12.7} \mathrm{Ga}_{1.3}$ compound has also been measured and the results are shown in Fig. 3. This compound is an antiferromagnet with a Néel temperature of $144^{\circ} \mathrm{C} .{ }^{12}$ The anomaly at the ordering temperature clearly indicates that this compound also shows a significant spontaneous magne-

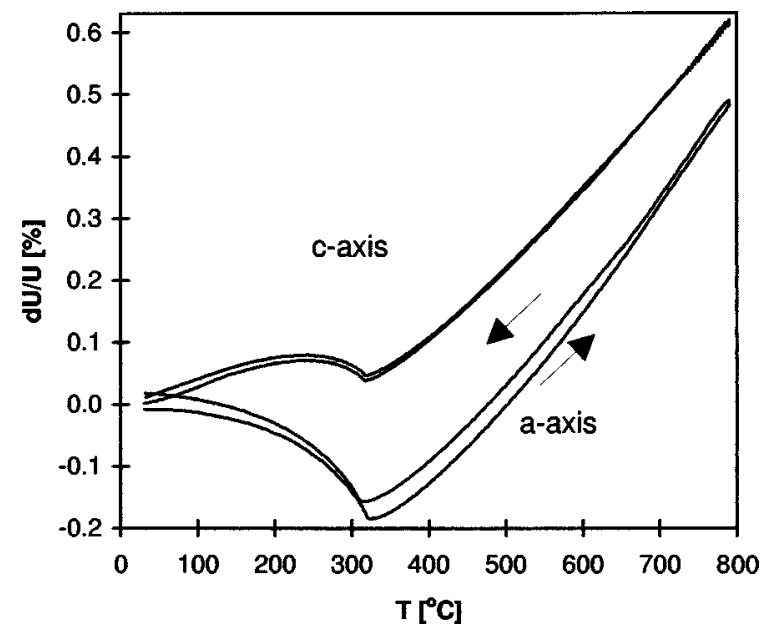

FIG. 2. Thermal expansion with increasing and decreasing temperature of an aligned $\mathrm{Nd}-\mathrm{FeB}$ magnet with a small amount of $\mathrm{Cu}$ additions measured along the $a$ and $c$ axes. Scan rate is $5^{\circ} \mathrm{C} / \mathrm{min}$.

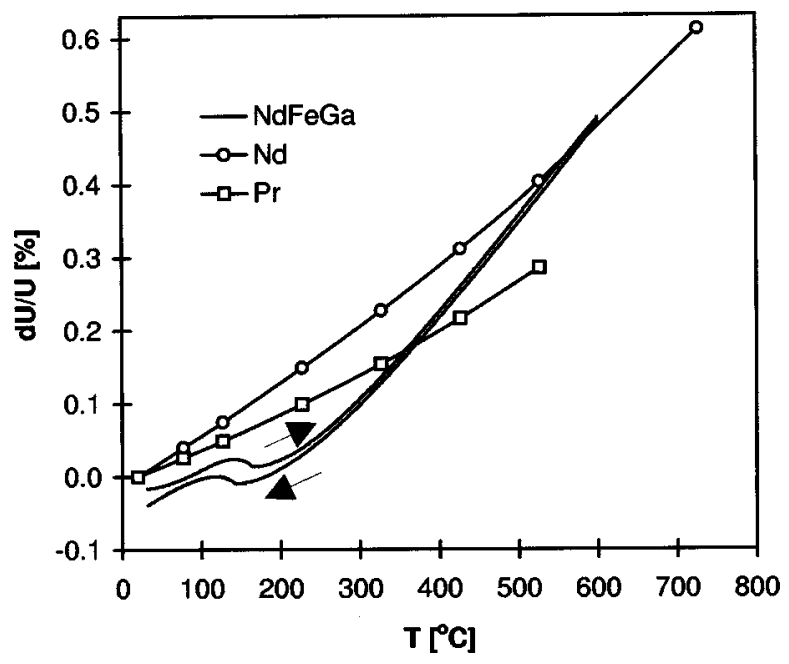

FIG. 3. Thermal expansion with increasing and decreasing temperature of a polycrystalline bulk piece of $\mathrm{Nd}_{6} \mathrm{Fe}_{12.7} \mathrm{Ga}_{1.3}$. Scan rate is $5{ }^{\circ} \mathrm{C} / \mathrm{min}$. Literature values of the thermal expansion of $\mathrm{Pr}$ and $\mathrm{Nd}$ are also plotted.

tostriction. In the same curve the expansion of $\mathrm{Nd}$ and $\mathrm{Pr}$, taken from Ref. 13, is plotted. Although the Nd- or Pr-rich intergranular phases have a slightly different composition than the respective elements, a similar thermal expansion is expected. From these figures, it can be seen that the difference in thermal expansion between the $a$ axis of the $\mathrm{Nd}_{2} \mathrm{Fe}_{14} \mathrm{~B}$ grains on the one hand and the $c$ axis of the $\mathrm{Nd}_{2} \mathrm{Fe}_{14} \mathrm{~B}$ grains or the intergranular phases on the other hand, is rather large. When cooling down, the misaligned $\mathrm{Nd}_{2} \mathrm{Fe}_{14} \mathrm{~B}$ grains and the intergranular grains will, therefore, exert a large stress on the $a$ axis of the $\mathrm{Nd}_{2} \mathrm{Fe}_{14} \mathrm{~B}$ grains.

In the calculation in the next paragraph, it is assumed that the stress on the grains is compressive. This is not $a$ priori true but depends on the percolation of the matrix phase. It is likely that both compressive and tensile stresses do exist, but only the compressive stress is important as coercivity in NdFeB magnets is determined by the areas of lowest anisotropy. By applying external pressure to the magnet an unambiguous compressive stress is induced. We used the negative magnetostriction of $\mathrm{Nd}_{2} \mathrm{Fe}_{14} \mathrm{~B}$ to make a pressure cell as displayed in Fig. 4. A hollow stainless-steel cylinder with a solid bottom and inner diameter of $d_{\mathrm{ss}}$ $=3.511(2) \mu \mathrm{m}$ was machined and a cylindrical $\mathrm{NdFeB}(\mathrm{Cu})$

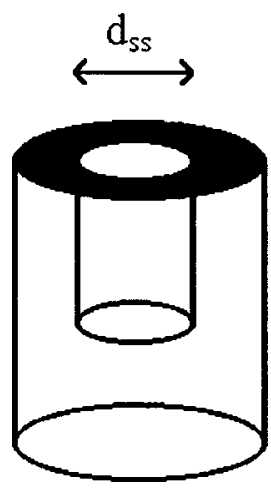

FIG. 4. Hollow stainless-steel cylinder used to exert pressure in the $a$ direction of the cylindrical $\mathrm{NdFeB}(5 \mathrm{wt} \%$ DyGa) magnet. 
magnet was ground to a diameter of $d_{\Phi}=3.521(1) \mu \mathrm{m}$. Both parts were heated to $300^{\circ} \mathrm{C}$ and the expansion of the stainless-steel cylinder and the shrinkage of the magnet in the $a$ direction made the magnet fit in the cylinder at that temperature. The experimental demagnetizing curves show a room-temperature coercivity which decreased slightly from $824(5)$ to $806(5) \mathrm{kA} / \mathrm{m}$. It should be noted that the small magnetization of the stainless steel has a negligible influence on the field experienced by the magnet.

\section{CALCULATIONS}

It is important to note that the penetration depth of the stress is much larger than the depth in which a reversed domain is nucleated. Street, Day, and Dunlop ${ }^{14}$ have measured the nucleation volume of a $\mathrm{Nd}_{2} \mathrm{Fe}_{14} \mathrm{~B}$ grain and derived a value of approximately $5 \times 10^{-25} \mathrm{~m}^{3}$, which is for a cubic nucleation volume equal to a depth of $8 \mathrm{~nm}$, roughly twice the domain-wall width $\delta_{b}$. As the nucleation always happens at the grain boundary, the coercivity is only determined by the anisotropy in the stressed region. It is, therefore, likely that stress causes a reduced anisotropy at the grain boundaries, and hence, may explain the large difference in coercivity between magnets with and without the $\delta$ phase. The idea of stress influencing the coercivity of $\mathrm{NdFeB}$ magnets has been put forward by Ramesh, ${ }^{5}$ and this calculation will progress along the same line. However, a number of essential elements will be changed. First, the magnetization-dependent magnetostriction coefficients will be used, whereas Ramesh has used spontaneous magnetostriction coefficients. Second, the compressibility of the intergranular phase will be taken into account. Third, the difference in thermal expansion will be calculated from room temperature to the melting point of the intergranular phase.

The magnetoelastic anisotropy constant is expressed in terms of the strain on the $\mathrm{Nd}_{2} \mathrm{Fe}_{14} \mathrm{~B}$ grains in Eq. (4). The strain can easily be calculated from the differences in the thermal expansion of the various phases when the magnet is cooled down after sintering. However, the Nd-rich phase is still liquid in the first stage of cooling, and it is assumed that during this cooling, no stress will occur. Only when the temperature falls below the melting temperature of the Nd-rich phase $T_{m}$, stresses will be induced. The total strain difference $\Delta U_{a}\left(T_{m}\right)$ upon cooling down from $T_{m}$ can be read from Figs. 2 and 3. Because the Nd-rich phase is "less stiff", than the $\mathrm{Nd}_{2} \mathrm{Fe}_{14} \mathrm{~B}$ phase, most of the strain will be incorporated in the Nd-rich phase. This results in a strain of the $\mathrm{Nd}_{2} \mathrm{Fe}_{14} \mathrm{~B}$ grains, which differs significantly from $\Delta U_{a}\left(T_{m}\right)$. To calculate the strain in the $\mathrm{Nd}_{2} \mathrm{Fe}_{14} \mathrm{~B}$ grains, the effective two-grain Young's modulus must be used. For a situation as in Fig. 1(a), the intergranular phase is of the same size as the stressed region in the $\mathrm{Nd}_{2} \mathrm{Fe}_{14} \mathrm{~B}$ grains. The strain in the $\mathrm{Nd}_{2} \mathrm{Fe}_{14} \mathrm{~B}$ grain along the $a$ direction is then given by

$$
\epsilon_{a}=\Delta U_{a}\left(T_{m}\right) \frac{E(I)}{E(\Phi)+E(I)},
$$

with $E(\Phi)$ the Young's modulus of the $\mathrm{Nd}_{2} \mathrm{Fe}_{14} \mathrm{~B}$ grains and $E(I)$ the Young's modulus of the intergranular phase. The anisotropy due to the difference in the thermal expansion of the Nd-rich phase and the $a$ axis of the $\mathrm{Nd}_{2} \mathrm{Fe}_{14} \mathrm{~B}$ grains can be calculated using $T_{m}=640{ }^{\circ} \mathrm{C}$ and the data for the magnetostriction and Youngs moduli collected in Table I. This gives a strain of $\Delta U_{a}\left(T_{m}\right)=3.2 \times 10^{-3}$ and $\epsilon_{a}=6.9 \times 10^{-4}$. In addition, Eq. (4) and the approximations for the stiffness coefficients from Table I, result in a magnetoelastic anisotropy of $K_{\text {m.e. }}=24 \mathrm{~kJ} / \mathrm{m}^{3}$. This value is much lower than the value found by Ramesh, ${ }^{5}$ since the anisotropic magnetostrictions are much smaller than the spontaneous magnetostrictions and the compressibility of the intergranular phase is rather high. More important, the value for the magnetoelastic anisotropy is negligible compared to the magnetocrystalline anisotropy of $K_{\text {m.c. }}=4.9 \mathrm{MJ} / \mathrm{m}^{3}$. For other intergranular phases similar calculations can be made and, although the Young's modulus of the $\mathrm{Nd}_{6} \mathrm{Fe}_{12.7} \mathrm{Ga}_{1.3}$ is unknown, it can be assessed that the magnetoelastic anisotropy will be of the same order of magnitude, i.e., considerably smaller than the magnetocrystalline anisotropy.

A compressive stress is exerted on the magnet upon cooling in the stainless-steel cylinder. Its value can be easily calculated using Eq. (14) with $\Delta U_{a}=\left(d_{\Phi}-d_{\mathrm{ss}}\right) / d_{\mathrm{ss}}$. This corresponds to an external pressure of $1.8 \mathrm{kbar}$ in the $a$ direction of the magnet and leads to an expected decrease in coercivity of $56 \pm 18 \mathrm{~kJ} / \mathrm{m}^{3}$. This number is slightly larger than the experimental decrease of around $18 \pm 10 \mathrm{~kJ} / \mathrm{m}^{3}$. However, since both calculated and measured effects of magnetostriction on coercivity are so small, it is concluded that homogeneous magnetostriction effects have no substantial influence on the anisotropy of sintered $\mathrm{NdFeB}$ magnets, neither theoretically nor experimentally.

However, it cannot be excluded that more subtle processes of induced stress play an important role. Because the nucleation volume is much smaller than the observed strained region, inhomogeneous stress distribution should be taken into account. Lattice mismatch between the $\mathrm{Nd}_{2} \mathrm{Fe}_{14} \mathrm{~B}$ grains and the intergranular phases or thermally induced stresses at triple points or other irregularities may cause very large strains in regions of the size of the nucleation volume. Furthermore, due to missing atoms, and therefore, symmetry breaking at the grain surface, the magnetocrystalline anisotropy at the grain surface is entirely different from the bulk anisotropy. ${ }^{15}$ However, no estimate of the size of these effects can be made. Therefore, it has to be concluded that a plausible explanation for the strong difference in coercivity between magnets with and without the $\delta$-intergranular phase is still missing.

\section{CONCLUSIONS}

An expression relating stress and magnetoelastic anisotropy has been derived for tetragonal compounds. It is shown that the orthorhombic distortion always lowers the anisotropy in tetragonal compounds no matter whether the stress is tensile or compressive. The stress in the $\mathrm{Nd}_{2} \mathrm{Fe}_{14} \mathrm{~B}$ grains that is experimentally observed by TEM is elastic and of local character near the grain boundary (penetration depth $\sim 200 \mathrm{~nm}$ ). However, the calculations show that in sintered $\mathrm{NdFeB}$ permanent magnets, the average anisotropy induced by the difference in thermal expansion of the various phases 
is small compared to the magnetocrystalline anisotropy. This is experimentally confirmed by the observation that applying a compressive stress of $1.8 \mathrm{kbar}$ has no significant influence on the coercivity of $\mathrm{NdFeB}(\mathrm{Cu})$ magnets. Because the nucleation volume of reversed domains is much smaller than the stressed region, inhomogeneous stress within the stressed region may determine coercivity when it is assumed that the stress varies over more than one order of magnitude.

\section{ACKNOWLEDGMENTS}

The authors would like to thank M. J. Verheijen for TEM measurements and K. H. J. Buschow and F. R. de Boer for valuable discussions. This research has been supported by the Dutch Technology Foundation (S.T.W.).

${ }^{1}$ J. F. Herbst, Rev. Mod. Phys. 63, 819 (1991).

${ }^{2}$ K. G. Knoch, A. Kianvash, and I. R. Harris, IEEE Trans. Magn. 28, 2142 (1992).
${ }^{3}$ C. H. de Groot, K. H. J. Buschow, F. R. de Boer, and K. de Kort, J. Appl. Phys. 83, 388 (1998).

${ }^{4}$ H. Kronmüller, K. -Dürst, and M. Sagawa, J. Magn. Magn. Mater. 74, 291 (1988).

${ }^{5}$ R. Ramesh, J. Appl. Phys. 68, 5767 (1990); 68, 5772 (1990).

${ }^{6}$ S. Chikazumi, Physics of Magnetism (Krieger, Malabar, FL, 1978).

${ }^{7}$ P. Morin and D. Schmitt, in Ferromagnetic Materials, edited by K. H. J. Buschow and E. P. Wohlfarth (North-Holland, Amsterdam, 1990), Vol. 7, Chap. 1.

${ }^{8}$ M. D. Kuz'min and J. M. D. Coey, Phys. Rev. B 50, 12533 (1994).

${ }^{9}$ C. Rudowicz, J. Phys. C 18, 1415 (1985).

${ }^{10}$ P. A. Algarabel, M. R. Ibarra, C. Marquina, A. del Moral, and S. Zemiri, J. Magn. Magn. Mater. 84, 109 (1990).

${ }^{11}$ H. Fujii, H. Nagata, Y. Uwatoko, T. Okamoto, H. Yamamoto, and M. Sagawa, J. Magn. Magn. Mater. 70, 331 (1987).

${ }^{12}$ C. H. de Groot, K. H. J. Buschow, and F. R. de Boer, Phys. Rev. B 57, 11472 (1998).

${ }^{13}$ Handbook of Chemistry and Physics, 68th ed., edited by R. C. Weast (CRC, Berlin, 1988).

${ }^{14}$ R. Street, R. K. Day, and J. B. Dunlop, J. Magn. Magn. Mater. 69, 106 (1987)

${ }^{15}$ L. Néel, Compt. Rend. 237, 1468 (1953). 